\title{
Virtual simulation experiment in the course Laser Principles and Techniques for undergraduates
}

\author{
Kun Li, Bo Wu
}

Kun Li, Bo Wu, "Virtual simulation experiment in the course Laser Principles and Techniques for undergraduates," Proc. SPIE 10452, 14th Conference on Education and Training in Optics and Photonics: ETOP 2017, 104525P (16 August 2017); doi: 10.1117/12.2269784

SPIE Event: 14th Conference on Education and Training in Optics and Photonics, ETOP 2017, 2017, Hangzhou, China 


\title{
Virtual simulation experiment in the course Laser Principles and
}

\section{Techniques for undergraduates}

\author{
Li Kun ${ }^{* a}$, Wu Bo ${ }^{\text {b }}$ \\ ( a. School of Optoelectronic Information, University of Electronic Science and Technology of \\ China, Chengdu, China; b. College of Optoelectronic technology, Chengdu University of \\ Information Engineering, Chengdu, China )
}

\begin{abstract}
The course Laser Principle and Technology for undergraduates is a multi-physics subject with main contents of laser's basic principle, laser modulation techniques, Q-switching techniques, etc. . In order to help students understand the complex theory and to integrate the theory with the engineering practice, we developed a virtual simulation platform/software. This platform consists of three main modules (laser generation, laser propagation and laser controlling), which can be subdivided into eight secondary modules, including laser output characteristics, laser resonator, laser modulation, frequency conversion, et al. . Each module has its input and output parameters and can be modified by the user. The theoretical models and the algorithms are introduced in this article. The output characteristics of the relaxation oscillation process are presented as an example of the simulation results.
\end{abstract}

Keywords: Virtual simulation experiment ; relaxation oscillations; rate equations; Runge-Kutta method

\section{INTRODUCTION}

The course Laser Principles and Technology is an integrated curriculum for undergraduates, related with multiple basic subjects such as quantum mechanics, statistical physics, electromagnetic field theory, physical optics and applied optics. The purpose of this course is basically to answer the following questions: how does the laser generate; why laser has high brightness, small divergence angle and high coherence; how to increase the laser intensity, compress the pulse width, change the frequency, et al. . The main contents of this course include the interaction of light with materials, the emission line width and broadening, the laser cavities modes, the propagation of the Gaussian beams, the Q-switching and mode locking techniques, etc. . In order to help the students understand the abstract physical concepts and to integrate the theory with the engineering practice, we developed a virtual simulation experiment platform/software, namely Laser V-Lab 1.0, to provide virtual simulation experiments about the key knowledge in this course.

The main GUI and the module structure of the software Laser V-Lab 1.0 are shown in Fig. 1. The software contains three main modules (laser generating, laser propagation and laser controlling), which can be subdivided into the following eight secondary modules:

1) Laser Output Characteristics Module. The transient laser output characteristics and the relaxation oscillation process in different laser gain mediums, such as Nd:YAG[1], Ti: Sapphire[2] and Ruby[3], can be simulated in this module. The fourth-order Runge-Kutta method is used to provide numerical solution of the rate equations with sufficient accuracy.

2) Optical Resonators Module. In this module, the stability of the passive optical resonator cavity can be analyzed, given the cavity structure and parameters. The establishment of laser transverse mode can be simulated by using Fox-Li *1k76@foxmail.com

14th Conference on Education and Training in Optics and Photonics: ETOP 2017, edited by Xu Liu, Xi-Cheng Zhang, Proc. of SPIE Vol. 10452, 104525P - (c) 2017 ICO, IEEE, OSA, SPIE CCC code: $0277-786 \mathrm{X} / 17 / \$ 18 \cdot$ doi: $10.1117 / 12.2269784$

Proc. of SPIE Vol. 10452 104525P-1 
numerical iterative method[4] and Fresnel Kirchhoff diffraction integral algorithm[5], respectively.

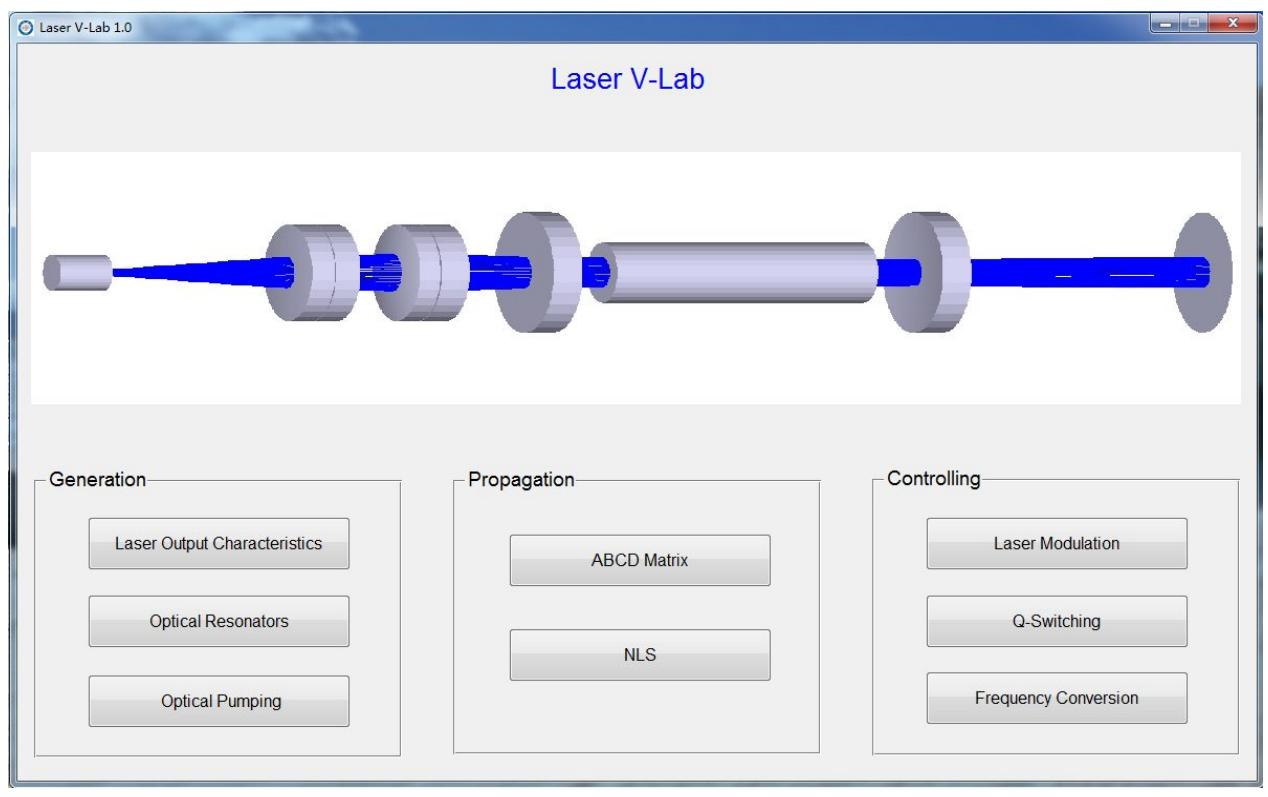

Fig. 1. The main GUI and the module structure of the software Laser V-Lab 1.0

3) Optical Pumping Module. Analyzing the distribution of pump light in the gain medium by using ray tracing method[6].

4) ABCD matrix Module. Simulating the transformation of the fundamental mode Gaussian beam in laser resonator or in space with $A B C D$ matrix[7].

5) NLS Module. Analyzing the diffraction, the group velocity, group velocity dispersion, diffraction and self focusing effect of the Gaussian beam by solving the Nonlinear Schrödinger equation with split-step Fourier transform $\operatorname{method}[8,9]$.

6) Laser Modulation Module. Three types of laser modulation techniques including electro-optical, acousto-optical and magneto-optical modulation can be simulated.

7) Q-Switching Module. The influence of the pumping power, the parameters of the gain medium and the laser resonator on the power and the pulse width of the output laser can be simulated by numerically solution of the Q-switching rate equations[10].

8) Nonlinear Frequency Conversion Module. In this module, the coupled wave equations of the nonlinear frequency conversion process are calculated by using the split-step Fourier transform and the fourth-order Runge-Kutta method. The factors, that influence the conversion efficiency, the pulse with and the peak intensity of the harmonic laser, can be analyzed. These factors include the peak intensity and the pulse width of the fundamental light, the detuning angle and the length of the nonlinear crystal[11].

Each of the above mentioned modules has its own interface where the user can specify and modify the input and output parameters.

\section{SIMULATION EXAMPLE}

The figure 2 shows the interface of the Laser Output Characteristics Module. We take $\mathrm{Nd}^{3+}$ : YAG as an example to 
illustrate its transient output characteristics and to explain the relaxation oscillation process. The rate equations[12, 13] is given in the top left corner of the interface (Fig.2) and also in Eqs.1.

$$
\begin{aligned}
& \left\{\begin{array}{l}
\frac{d \Phi}{d t}=\frac{\Phi}{t_{r}}\left\{2 \sigma \Delta n l_{g}-\left[\ln \frac{1}{R}+2 a_{s c} l_{g}\right]\right\} \\
\frac{d \Delta n}{d t}=R_{p}-\sigma c \Phi \Delta n-\frac{\Delta n}{t_{g}}
\end{array}\right. \\
& R_{p}=\frac{P \lambda}{\pi h c w_{p}{ }^{2} l_{g}}
\end{aligned}
$$

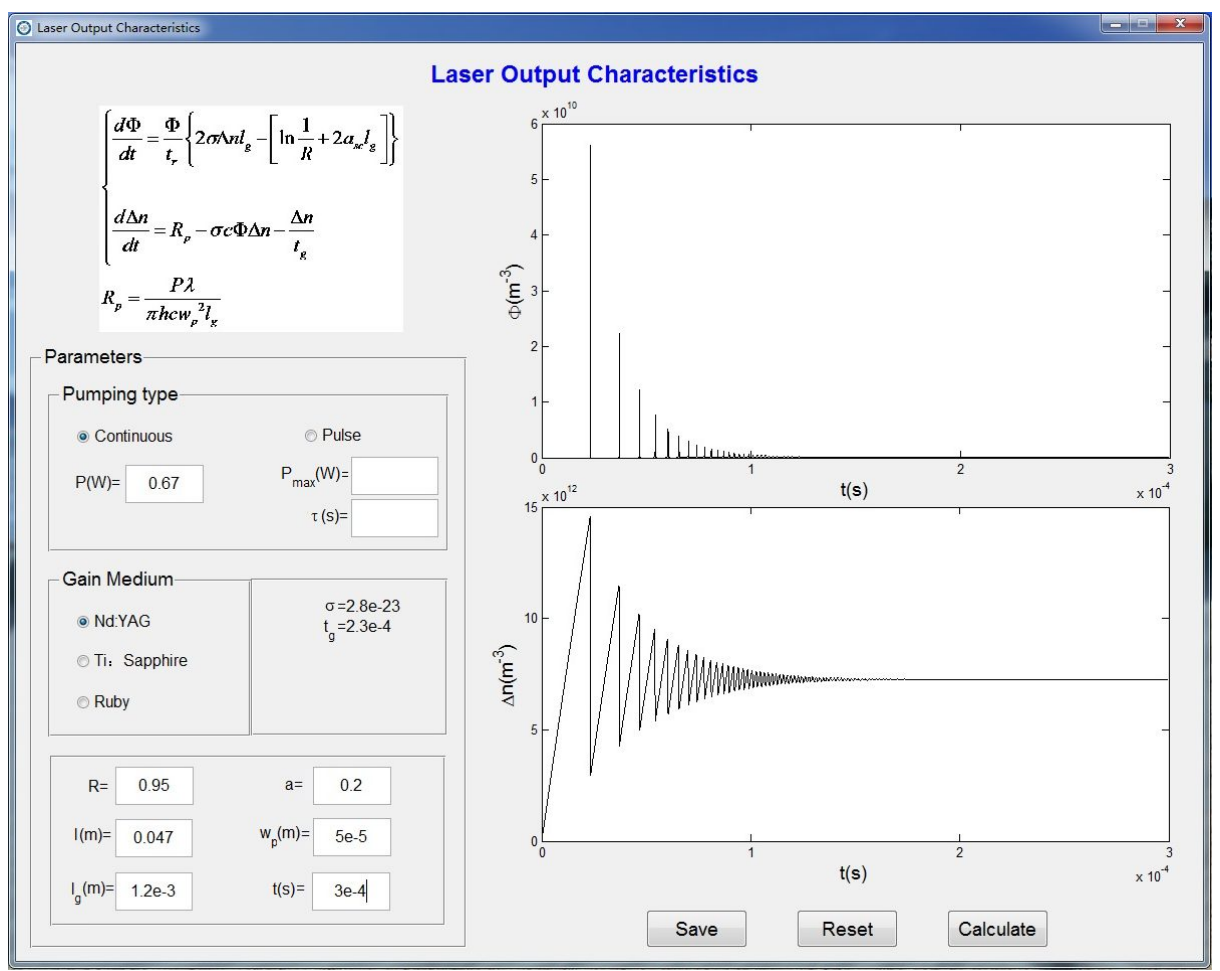

Fig.2 The interface of the Laser Output Characteristics Module

The physical meaning of the parameters are as following[12, 13]:

$\Delta n$ : The population inversion density of the gain medium;

$\Phi$ : The photon density in the laser cavity;

$\sigma$ : The stimulated emission cross section area of the gain medium;

$\lambda$ : The wavelength of the pumping light;

$t_{g}$ : The lifetime of the upper laser level of the gain medium

$R$ : The reflectivity of the output mirror;

$\alpha_{s c}$ : The scattering loss;

$t_{r}$ : The cavity round-trip time; $t_{r}=L / \mathrm{c}$, where $L$ is the optical length of the resonator, $\mathrm{c}$ is the speed of light;

$l_{g}$ : The length of the laser gain medium; 
$R_{p}$ : The volumetric pump rate into the upper laser level;

$w_{p}$ : The spot radius of the pumping light;

$P$ : The pump power absorbed by the gain medium;

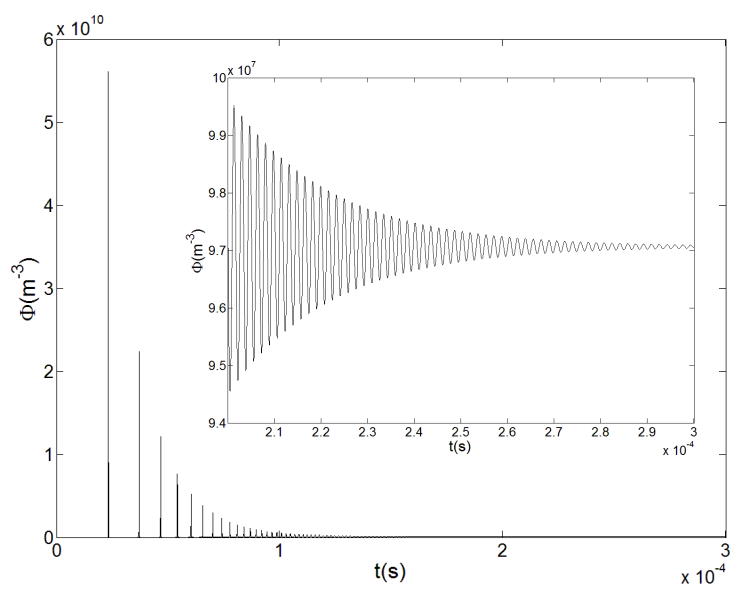

Fig.3 The evolution of the photon density in the laser cavity

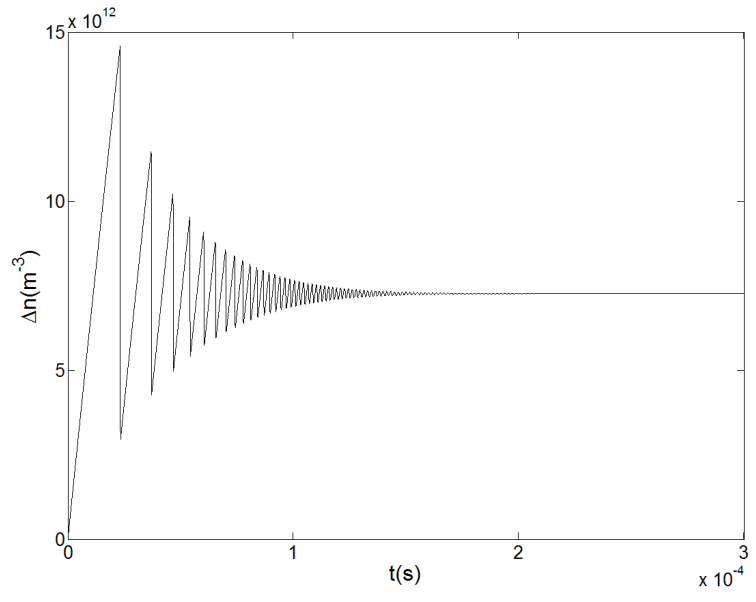

Fig.4 The evolution of the population inversion density of the gain medium

The calculation result can also be saved as JPG files as Fig.3 and Fig.4, which illustrated the evolution of the photon density in the laser cavity and the population inversion density of the gain medium on time scale, respectively. We can see from Fig. 3 that the photon density oscillated intensively in the beginning $0.2 \mathrm{~ms}$ and then turned to be stable in the next $0.1 \mathrm{~ms}$. This reveals the transition from the relaxation oscillation to stable state under the continuous pumping. The population inversion density of the gain medium oscillated around its threshold and trended to be stable at its threshold after $0.2 \mathrm{~ms}$.

In most teaching material about the course laser principles, the output characteristics of the laser is analyzed based on analytical calculation under the following two conditions: a) short pulse pumping, i.e. the pumping pulse width $t_{p}<<$ the lifetime of the upper laser level of the gain medium $t_{g}$; and b) long pulse pumping or continuous pumping, i.e. $t_{p}>>t_{g}$. As for the relaxation oscillation state, the time dependent perturbation theory is often used to give approximation solutions of the rate equations. These calculation results have the limitations that they cannot be very accurate compared with the real laser system.

The calculation accuracy can be obviously improved with numerical method. The parameters such as the pumping rate, the cavity loss, the population inversion density of different laser medium, can be specified or modified by the user to see their influences on the output behavior of the laser. These simulations in this module of our software can help students better understand the free oscillation laser in the actual engineering application.

\section{CONCLUSIONS}

By using this virtual simulation experiment software Laser V-Lab, students can have more intuitive understanding of the structures and the functions of the laser device, as well as better comprehension about the concepts and the variables in the physical formulas of the course. The virtual experiment can be a necessary complement to curricular study and can play an important role to encourage the students' creativity. 
At present, the simulation modules in this software are aimed at the curriculum of Laser Principles and Techniques. The upgraded software will further increase its interactivity with the user, improve the animation effect, and add some new functional modules such as to analyze the thermal effect in the gain medium, to analyze the beam quality, to add more types of laser medium, etc. .

\section{ACKNOWLEDGEMENTS}

This work was supported by the teaching reform projects for undergraduates of the University of Electronic Science and Technology of China (Project Number: 2017XJYJ-ZD12).

\section{REFERENCES}

[1] Ghani B. A., Abbas B., Sheikh-Khalil M., "Mathematical modeling of the intracavity Q-switched Nd-glass laser," Optics and Laser Technology, 37 (2), 117-124 (2005).

[2] Song F., Yao J. Q., Zhou D. W., "Rate-equation theory and experimental research on dual-wavelength operation of a Ti : sapphire laser," Applied Physics B-Lasers and Optics, 72(5), 605-610 (2001).

[3] Soukieh M., Ghani B. A., Hammadi M., "Numerical calculations of intracavity dye Q-switched ruby laser," Optics and Lasers in Engineering, 41(1), 177-187 (2004).

[4] Zou H., Zhou L. F., Yang Z., "Simulation study on transverse mode of laser resonator," Proc. SPIE 9656, (2015).

[5] Morin M., Belanger P. A., "Diffractive analysis of annular resonators," Applied Optics, 31(12), 1942-1947 (1992).

[6] Asoubar D., Zhang S., Kuhn M., "Laser resonator modeling by field tracing: a flexible approach for fully vectorial transversal eigenmode calculation," Journal of the Optical Society of America B-Optical Physics, 33(11), 2565-2573 (2014).

[7] Su Binghua, Xue Junwen, Sun Lu, "Generalised ABCD matrix treatment for laser resonators and beam propagation," Optics and Laser Technology, 43(7), 1318-1320 (2011).

[8] Shao Jing, Liang Xiaojun, Kumar Shiva, "Comparison of split-step Fourier schemes for simulating fiber optic communication systems,” IEEE Photonics Journal, 6(4), 7200515 (2014).

[9] Cambronero-Lopez F., Gomez-Varela A. I., Bao-Varela C. "Designing an ultrafast laser virtual laboratory using MATLAB GUIDE,” European Journal of Physics, 38(3), 034006 (2017).

[10] Li Q., Feng B., Zhang D., "Numerical solution and experiment of a self-Q-switched $946 \mathrm{~nm} \mathrm{Cr,} \mathrm{Nd:YAG}$ laser," Applied Physics B-Laser and Optics", 93(2-3), 421-427 (2008).

[11] Li Kun, Zhang Bin, "Analysis of broadband third harmonic generation with non-collinear angular dispersion in KDP crystals," Optics Communications, 281(8), 2271-2278 (2008).

[12] Zhao Jia, Zhao Shengzhi, Li Kang, "Optimization of passively Q-switched and mode-locked laser with $\mathrm{Cr}^{4+}$ :YAG saturable absorber," Optics Communications, 284 (6),1648-1651 (2011).

[13] Dong J., Deng P. Z., "Laser performance of monolithic Cr, Nd: YAG self-Q-switched laser," Optics Communications, 220, 425-431 (2003). 